\title{
Plasma metabolomics and proteomics profiling after a postprandial challenge reveal subtle diet effects on human metabolic status
}

\author{
Linette Pellis • Marjan J. van Erk • Ben van Ommen • Gertruud C. M. Bakker • \\ Henk F. J. Hendriks • Nicole H. P. Cnubben - Robert Kleemann • Eugene P. van Someren • \\ Ivana Bobeldijk $\cdot$ Carina M. Rubingh $\cdot$ Suzan Wopereis
}

Received: 31 March 2011/ Accepted: 12 May 2011/Published online: 28 May 2011

(C) The Author(s) 2011. This article is published with open access at Springerlink.com

\begin{abstract}
We introduce the metabolomics and proteomics based Postprandial Challenge Test (PCT) to quantify the postprandial response of multiple metabolic processes in humans in a standardized manner. The PCT comprised consumption of a standardized $500 \mathrm{ml}$ dairy shake containing respectively 59, 30 and 12 energy percent lipids, carbohydrates and protein. During a $6 \mathrm{~h}$ time course after PCT 145 plasma metabolites, 79 proteins and 7 clinical chemistry parameters were quantified. Multiple processes related to metabolism, oxidation and inflammation reacted to the PCT, as demonstrated by changes of 106 metabolites, 31 proteins and 5 clinical chemistry parameters. The PCT was applied in a dietary intervention study to evaluate if the PCT would reveal additional metabolic changes compared to non-perturbed conditions. The study consisted of a 5-week intervention with a supplement mix of antiinflammatory compounds in a crossover design with 36 overweight subjects. Of the 231 quantified parameters, 31 had different responses over time between treated and control groups, revealing differences in amino acid
\end{abstract}

Electronic supplementary material The online version of this article (doi:10.1007/s11306-011-0320-5) contains supplementary material, which is available to authorized users.

L. Pellis $(\bowtie) \cdot$ M. J. van Erk · B. van Ommen ·

G. C. M. Bakker - H. F. J. Hendriks .

N. H. P. Cnubben · E. P. van Someren .

C. M. Rubingh $\cdot \mathrm{S}$. Wopereis

TNO, PO Box 360, 3700 AJ Zeist, The Netherlands

e-mail: linette.pellis@tno.nl

R. Kleemann

TNO, PO Box 2215, 2301 CE Leiden, The Netherlands

I. Bobeldijk

TNO, Triskelion, PO Box 360, 3700 AJ Zeist, The Netherlands metabolism, oxidative stress, inflammation and endocrine metabolism. The results showed that the acute, short term metabolic responses to the PCT were different in subjects on the supplement mix compared to the controls. The PCT provided additional metabolic changes related to the dietary intervention not observed in non-perturbed conditions. Thus, a metabolomics based quantification of a standardized perturbation of metabolic homeostasis is more informative on metabolic status and subtle health effects induced by (dietary) interventions than quantification of the homeostatic situation.

Keywords Postprandial challenge - Metabolic profiling · Proteomic profiling · Plasma

\section{Introduction}

The physiological and biochemical response to a dietary perturbation is complex. It includes energy storage mostly orchestrated by insulin and involves metabolic switches in several organs like liver, muscle and adipose tissue, accompanied by several compensating processes such as inflammation and oxidative stress (Carroll and Schade 2003; Esposito et al. 2003; Nappo et al. 2002; Neri et al. 2005; Wybranska et al. 2003). Many of these processes share pathways and control mechanisms that are common to metabolic, inflammatory and oxidative stress processes. For instance, the response to a "simple" glucose bolus involves modulation of glucose itself, triglycerides, blood pressure, cholesterol, inflammation and oxidation (Nakatsuji et al. 2010).

The postprandial response depends on, and involves multiple factors. Multiple processes related to metabolism, inflammation and oxidation are affected (Lundman et al. 2007). The type, nature and amount of fat influences lipid 
clearance, just like the amount and type of carbohydrate, protein, fiber and alcohol in a meal (Cianflone et al. 2008; Lopez-Miranda et al. 2007). Gender, genetics, age, body size, exercise and weight loss influence postprandial metabolism (Lopez-Miranda et al. 2007; Paglialunga and Cianflone 2007), as do various metabolic syndrome related conditions (Ghanim et al. 2009; Lopez-Miranda et al. 2007; Paglialunga and Cianflone 2007). Increasing knowledge on molecular aspects of the postprandial response is becoming available. Plasma metabolome changes were reported during an oral glucose tolerance test (OGTT) (Shaham et al. 2008; Wopereis et al. 2009; Zhao et al. 2009), similarly, the plasma lipidome response was assessed during a lipid challenge (Zivkovic et al. 2008). Intake of a high fat meal alters metabolism and increases inflammation and oxidative stress, thereby reducing amongst others vascular function (Devaraj et al. 2008; Ghanim et al. 2009; Tsai et al. 2004). A high fat meal increases pro-inflammatory cytokines, such as plasma interleukin 6 (IL6) and 18 (IL18), factor VII, tumor necrosis factor alpha (TNF- $\alpha$ ), intercellular adhesion molecule-1 (ICAM-1) and vascular cell adhesion molecule-1 (VCAM-1) concentrations (Esposito et al. 2003; Lopez-Miranda et al. 2007; Lundman et al. 2007; Nappo et al. 2002). Saturated fatty acids negatively affect endothelial function, whereas monounsaturated fatty acids have neutral or modest beneficial effect on endothelial function, and polyunsaturated fatty acids have a positive effect (Jackson et al. 2007; Margioris 2009). Several oxidation markers are increased after a high fat meal, like ROS generation by mononuclear cells and thiobarbituric acid reactive substance concentrations (Ghanim et al. 2009). Oxidative stress may be an important mechanism by which postprandial lipidemia alters vascular function (Devaraj et al. 2008; Ghanim et al. 2009).

In nutrition and health research, the concept of perturbation of homeostasis to quantify health related processes is advancing (Elliott et al. 2007; van Ommen et al. 2009). The postprandial response reveals multiple aspects of metabolic health that would not be apparent from studying the fasting (homeostatic) parameters. The emerging nutrigenomics technologies, specifically metabolomics and lipidomics (van Ommen et al. 2008), allow analysis of multiple aspects of the postprandial response including quantification of molecular changes related to metabolic flexibility, robustness of homeostatic mechanisms and adaptive responses.

In this study, first the postprandial challenge test (PCT) was used to quantify postprandial metabolic response using metabolomic and proteomic profiling on endogenous processes related to metabolism, inflammation and oxidative stress. Secondly the PCT concept was applied in a nutritional intervention study to evaluate if the PCT would reveal additional metabolic changes compared to non-perturbed conditions. In a 5 week crossover doubleblind placebo controlled intervention study overweight males were given anti-inflammatory dietary mix (AIDM) (Bakker et al. 2010). The postprandial response of 231 metabolites and proteins to a postprandial challenge was measured and compared to fasting (homeostatic) values of these metabolites and proteins.

\section{Materials and methods}

\subsection{Study design, execution, and analysis}

The execution and analytical methodologies of the nutritional intervention study has previously been described in detail (Bakker et al. 2010). In short, a series of dietary products selected due to anti-inflammatory properties (resveratrol, green tea extract, alpha-tocopherol, vitamin $\mathrm{C}, \mathrm{n}-3$ poly unsaturated fatty acids and tomato extract) were combined and supplemented to 36 healthy overweight men (BMI $25.6-34.7 \mathrm{~kg} / \mathrm{m}^{2}$ ) with mildly elevated C-reactive protein (CRP) levels (1.0-8.1 $\mu \mathrm{g} / \mathrm{l})$ in a double-blind, placebo controlled, crossover study with test treatment periods of 5 weeks. At the end of the intervention and control exposure, a $500 \mathrm{ml}$ postprandial (fat $58.7 \mathrm{E} \%$ ) dairy shake, including $300 \mathrm{ml}$ custard, $150 \mathrm{ml}$ cream cheese and $50 \mathrm{ml}$ whipping cream (nutritional values in Table 3) was given to study the postprandial response. After an overnight fast the subjects received a light standardized breakfast. After at least a $4 \mathrm{~h}$ period without food and drinks (except water) they were offered the postprandial shake. At time points $0 \mathrm{~h}$ (fasting condition), $1 \mathrm{~h}, 2 \mathrm{~h}, 3 \mathrm{~h}, 4 \mathrm{~h}$ and $6 \mathrm{~h}$ after the postprandial dairy shake, blood samples were collected and analyzed using GC-MS metabolic profiling (145 plasma metabolites), multiplex proteomics (79 plasma proteins) and a series of clinical chemistry analyses (glucose, insulin, total free fatty acids, total triglycerides, hsCRP, IL6, and TNF $\alpha$ ).

\subsection{Metabolic profiling}

The GC-MS method used for the measurement of a broad range of metabolites was identical to the GC-MS method reported (Koek et al. 2011) for liver samples. In this study, $100 \mu \mathrm{l}$ of plasma was used extracted and further derivatized.

For GC-MS, a total of 504 plasma samples were analyzed in 18 different batches. All the samples of one particular subject were analyzed within the same batch, with two subjects per batch. In principle, all the samples were prepared and injected once. All the samples from two randomly selected subjects (20 samples in total) were repeated in the final 19th batch of the study. The performance of the applied metabolic profiling platforms was 
controlled by the frequent analysis of the quality control sample (QC), and method performance was monitored by 10 internal standards (including analogs and $2 \mathrm{H}-$ and $13 \mathrm{C}$ labeled metabolites), as described previously (Koek et al. 2011; van der Kloet et al. 2009). Batches were only accepted if the relative standard deviation (RSD) of the peak area ratio for all internal standards was $<20 \%$ in all analyzed study and QC samples. The QC sample, prepared by pooling study samples, represents the biochemical diversity of the study samples and allows the calculation of the analytical precision for all metabolites measured. The QC samples were used to select the most suitable internal standard for each detected metabolite. This was done by calculating the relative standard deviation (RSD) for each metabolite after separate normalization for each internal standard. The internal standard giving the lowest RSD in all the QC samples was subsequently selected to normalize all the study samples. This procedure was described previously in more detail (van der Kloet et al. 2009). After normalization of the data with the most suitable internal standard for each detected metabolite (peak), the QC sample data were further used to correct systematic errors (e.g. batch to batch response differences and also trends within the batches) by a single point calibration model (van der Kloet et al. 2009). After the above mentioned data correction steps, the metabolites were only accepted if the RSD of the determined (relative) concentration in all the QC samples was $<20 \%$, unless large differences between test groups were observed. Additional quality control of the final dataset was performed by comparing the duplicate measurements of the two randomly selected subjects. The results were described by van der Kloet et al. 2009.

In the end, the GC-MS data set consisted of 145 metabolite peaks that passed quality control requirements (Supplemental Table 2). Metabolites were annotated by using an in-house metabolite database containing retention time information, MS spectra (electron impact ionization) of reference substances and metabolites previously identified by the interpretation of mass spectra based on structural similarities with the analyzed reference compounds or spectra published in the literature. The confidence of identification was $100 \%$, unless indicated otherwise. Because of the applied sample preparation, some metabolites can result in more than 1 peak, for example two different derivatization products. These different peaks were all labeled as such and reported individually. In this way it could be checked whether all signals originating from the same metabolite resulted in a similar PCT response, which was the case.

\subsection{Multiplex proteome analysis}

Plasma samples were sent to Rules-Based Medicine Inc. (Austin, USA) for measurement of the concentration levels of 124 proteins (HumanMAP). Data were available for 33 subjects. The so-called $80 \%$ rule (Bijlsma et al. 2006) was applied to retain only those proteins which have $80 \%$ or more values above the detection limit for at least one of the two test groups, resulting in retention of 79 out of the 124 variables (Supplemental Table 2). Values below the detection limit that remained in the truncated data set were replaced by a value set at half of the detection limit. Values for remaining samples that were not measurable on the standard curve for a specific protein were set at 0.1 times the detection limit for that protein.

\subsection{Clinical chemistry measurements}

Serum and EDTA-blood was collected for clinical chemistry tests. Serum glucose, and insulin were analyzed by immunoturbidimetric techniques and non-esterified fatty acids (NEFA) and total triglycerides were measured by an enzymatically (Boehringer-Mannheim, Mannheim, Germany) on an Olympus AU400 clinical chemistry analyzer (Olympus-Diagnostica Europe, Hamburg, Germany). $\mathrm{TNF} \alpha$, IL-6 and hsCRP were measured in the plasma samples. The analyses were performed using multiplex kits (high-sensitivity human cytokine premixed Lincoplex kit; Linco Research, St Charles, MO).

\subsection{Area under curve calculations}

For all parameters measured during the PCT incremental areas under or over the baseline were calculated using the first measurement $(\mathrm{t} 0)$ as reference. We used the term area under the curve (AUC) to refer to both values, which were delineated as negative AUC (AUC-) and positive AUC $(\mathrm{AUC}+)$. The sum of the areas under and over the baseline was defined as total AUC (AUCt). The time required to reach the highest observed plasma concentration was defined as $\mathrm{T}_{\max }$ and the time required to reach the lowest observed plasma concentration as $T_{\min }$. $T_{\max }$ was only used for parameters with a cluster 1 or 2 time profile (see results and cluster analysis in this section), $\mathrm{T}_{\min }$ was only used for parameters with a cluster 3 time profile (see results and cluster analysis in this section). $\mathrm{T}_{\max }$ or $\mathrm{T}_{\min }$ in the other clusters were considered to be not relevant, since these corresponded with the first or the last time point of the curve.

\subsection{Statistical analysis}

The data sets were analyzed using a two-way ANOVA on time and treatment effects. Earlier (Bakker et al. 2010), we reported on statistical analysis of GC-MS and protein profiling data on intervention differences using a repeatedmeasures ANOVA. Only when no significant interaction between intervention and time was found, main 
intervention effects averaged over time were investigated. In this paper, we focus on main time effects only.

The effect of intervention was analyzed by one-way ANOVA on the calculated AUC variables. Data were log transformed if necessary. In all statistical tests performed, the null hypothesis (no effect) was rejected at the 0.05 level of probability. A Bonferroni adjustment was used to correct for multiple comparisons. The SAS statistical software package (versions 8.2 and 9.1; SAS Institute Inc, Cary, NC) was used for statistical analysis.

For variables with significant differences in AUC parameters (AUC+, AUC- or AUCt), the differences versus t0 were calculated for each time point and each subject. Mean \pm SEM of these differences in placebo and AIDM group were visualized in graphs (Fig. 2 and Supplemental Fig. 1). The plasma parameters with significant differences between AIDM and placebo for one or more AUC variables, but without a significant effect of time were checked for a dynamic response by visual inspection.

\subsection{Cluster analysis}

Hierarchical clustering was performed on the time profiles of all parameters with a significant time effect to create groups of parameters exhibiting a similar response to the PCT. For the hierarchical clustering, Pearson's correlation was used as distance measure and complete linkage was used to define distance between clusters. The number of 6 clusters was chosen manually taking into account the difference in response and the number of plasma parameters. We wanted to end up with clusters clearly distinct in type of response and containing enough parameters $(n>3)$.

\subsection{Network analysis}

Network (Fig. 3) was built in MetaCore version 6.2 (GeneGo Inc., St Joseph, MI, USA), using option 'auto expand'. Ingenuity Pathway Analysis version 9.0 (Ingenuity System, Redwood City, CA, USA) was used to obtain the associated functions of the network(s) from the six clusters. These obtained functions are discussed in Sect. 3 below.

\section{Results and discussion}

\subsection{Quantification of the postprandial response}

Comprehensive targeted metabolomics and proteomics analysis of the response to a postprandial challenge revealed major metabolic changes. Plasma concentrations versus time profiles were determined for 145 metabolites, 79 proteins and 7 clinical chemistry parameters. ANOVA revealed a significant time effect for 106 metabolites, 31 proteins and 5 clinical chemistry parameters. These 142 metabolites, plasma proteins and clinical chemistry parameters covered a broad range of biological processes, from energy metabolism including carbohydrates, lipids and proteins to regulatory processes and responses related to oxidative and inflammatory stress. These responses are described in detail below.

The 142 plasma parameters were clustered into 6 discrete postprandial response time courses. Various types of time courses were observed: rapid (maximum change in plasma concentration within $1-2 \mathrm{~h}$ ) as well as slow (maximum response after $6 \mathrm{~h}$ ) with both decreasing and increasing plasma concentrations. Figure 1 shows these 6 different time course profiles and Supplemental Table 1 lists the metabolites and proteins grouped to these different time course profiles. The chemical structure of 11 of the 106 changed metabolites was not identified and these were therefore excluded from Table 1.

\subsubsection{Glucose and carbohydrate metabolism}

The PCT (containing 30 energy percent (E\%) carbohydrates) caused a temporary increase in plasma concentrations of glucose (cluster 1) and insulin (cluster 2). Similar responses were found for several metabolites associated with carbohydrate metabolism, such as sucrose, 4-deoxyglucose and citric acid cycle metabolites. Fructose and the gut hormones glucagon like protein 1-active (GLP1active), protein YY (PYY) and pancreatic polypeptide (PP) showed a similar time course to glucose (cluster 1 or cluster 2). Concentrations of the monosaccharides xylose and arabinose decreased over time in response to PCT (cluster 4). Insulin facilitates the transport of these sugars from blood across the cell membrane (Goldtein et al. 1953). Consistent with mannose being a non-insulin sensitive sugar, the plasma concentration of mannose increased at later time points after PCT (cluster 6).

Apolipoprotein CIII had a similar response to the PCT as glucose. This response may also be controlled by insulin, because the apolipoprotein CIII (APOC3) promoter contains an insulin responsive element and insulin has been shown to downregulate APOC3 expression (Waterworth et al. 2003).

\subsubsection{Amino acid metabolism}

Most plasma amino acids reacted very similarly to the PCT: a rapid increase which returns to baseline within 4-5 h (cluster 2, glutamic acid in cluster (1). These amino acids probably originated from proteins present in the PCT formulation (12 $\mathrm{E} \%$ protein). Urea showed a cluster 1 profile, suggesting a surplus of amino acids that is 
A
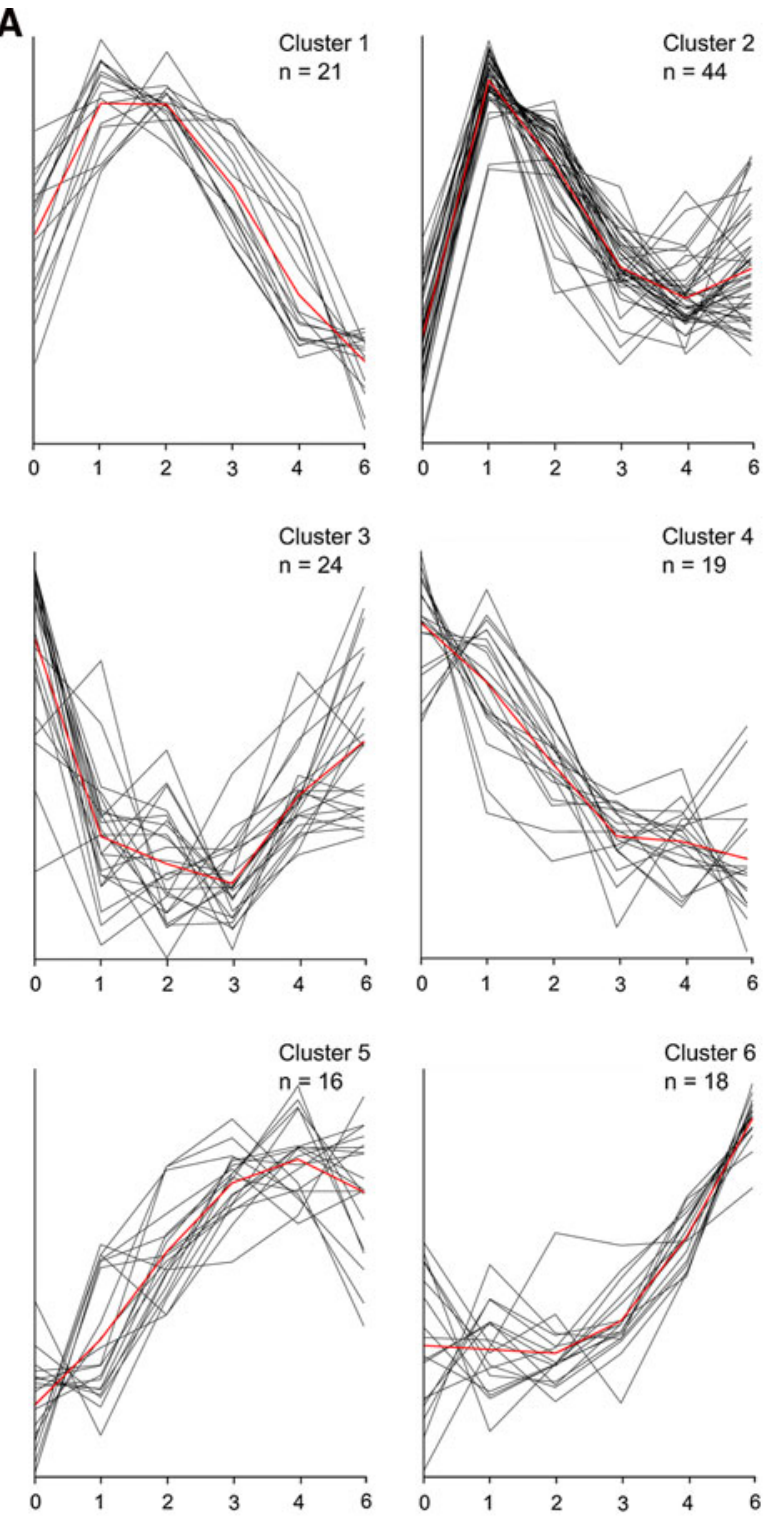

B

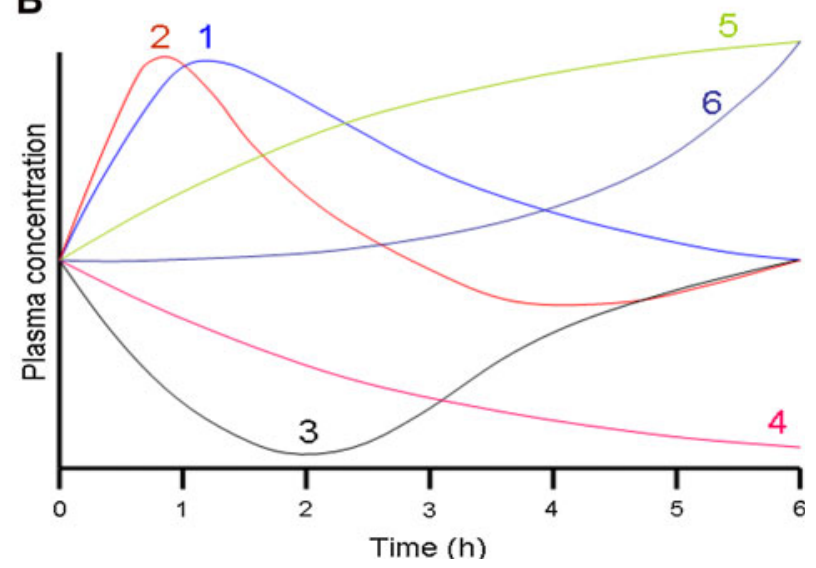

transaminated and deaminated for the production of glucose, fatty acids or energy. The amino acid derivatives 3-methylhistidine and creatinine both originate from
4Fig. 1 The six different observed postprandial time course profiles. a The time cluster profiles represented by the 142 different plasma metabolites and proteins with a significant effect of time. The red line represents the average cluster time profile. The $x$-axes were expressed as time (hours), the $y$-axes were expressed as relatively scaled concentrations. Time profile cluster 1 represented 21 plasma parameters with a classical absorption profile, reaching maximum values after $1-2 \mathrm{~h}$, followed by a continued reduction towards minimal values at the final $(6 \mathrm{~h})$ time point. Time profile cluster 2 , including 44 parameters, was similar to cluster 1 , with the main difference that parameters in cluster 2 reached minimum values around $4 \mathrm{~h}$ after postprandial challenge. Thus, the time profile clusters 1 and 2 mainly differ in the duration of the response ( 4 or $6 \mathrm{~h}$ after postprandial challenge). Time profile cluster 3 represented the parameters that decreased upon the PCT, with a subsequent recovery phase. The average time required to reach lowest plasma concentrations is $2-3 \mathrm{~h}$. This cluster contained 24 parameters with a significant time effect. Time profile cluster 4 (19 parameters) included parameters that steadily decreased during the $6 \mathrm{~h}$ time course. Time profile cluster 5 represented 16 plasma parameters that increased during most of the $6 \mathrm{~h}$ time course. The average time required to reach highest plasma concentrations was $\sim 4 \mathrm{~h}$. Finally, time profile cluster 6 included 18 parameters with a continuous increase in plasma concentration after an initial lag phase of approximately two hours. b The different time profile clusters summarized in one figure (Color figure online)

Table 1 Number of additionally identified changed parameters by applying the PCT and number of changed parameters by the dietary intervention at fasting $(\mathrm{t} 0)$ conditions

\begin{tabular}{lrlc}
\hline & $n$ & PCT (overlap fasting) & Fasting \\
\hline Metabolites & 145 & $18(12)$ & 48 \\
Proteins & 79 & $13(3)$ & 9 \\
Clinical chemistry & 7 & 0 & 1 \\
Total plasma parameters & 231 & $31(14)$ & 58 \\
\hline
\end{tabular}

muscle (van Eijk et al. 1990; Wyss and Kaddurah-Daouk 2000). Since these muscle metabolites showed a temporary plasma increase while the PCT contains dairy protein and no meat protein, these data suggest that the PCT also affects muscle metabolism.

\subsubsection{Bone metabolism}

Plasma hydroxyproline (and methyl-hydroxyproline) concentrations decreased in response to PCT (cluster 4). Hydroxyproline is mainly present in collagen, and is a marker for bone turnover, suggesting that bone metabolism may be influenced during postprandial metabolism (Minisola et al. 1985). In cluster 4 cortisol, MMP2, TNFRII, VCAM1 and uric acid all play a role in bone turnover and osteoarthritis (Walsh and Henriksen 2010; Schett et al. 2009; Nowatzky et al. 2010). Mutations in MMP2 and TNFRII diminish bone resorption (Cawston and Young 2010; Riches and Ralston 2010). This corresponds with the fact that bone resorption is immediately reduced after food intake (Parfitt 2002). 


\subsubsection{Lipid and lipoprotein metabolism}

The PCT formulation is rich in lipids (59 E\%). Total triglycerides, C10:0 free fatty acid, monoglycerides C16:0 and C18:1 levels rose immediately after intake (cluster 1), followed by slow increase in C12:0 and C14:0 free fatty acids (cluster 5). In the small intestine pancreatic lipases hydrolyse triglycerides into 2-monoacylglycerols and free fatty acids, which are re-esterified into triglycerides and liberated in the blood via chylomicrons. Fatty acids C10:0, C12:0 and C14:0 are poor substrates for re-esterification into triglycerides, because the specific medium-chain acyl-CoA synthase required for their activation is absent. Thus these fatty acids enter plasma directly as unesterified fatty acids (Frayn 2010). In addition, the monoglycerides 16:0 and 18:1 are released from chylomicrons during hydrolysis of triglycerides by lipoprotein lipase in adipose tissue. This process is stimulated by insulin (Wang and Eckel 2009), which also showed an immediate increase in response to the PCT.

Plasma concentrations of longer free fatty acids (C16:0, C16:1, C17:0, C18:0, C18:1, C18:2, arachidonic acid (C20:4)) and glycerol increased after a lag time (cluster 6). Insulin-immediately released after food intake- suppresses fat mobilization for energy production. In the late phase of the time course, adipose tissue triglycerides are likely to be hydrolyzed by hormone-sensitive lipase for beta-oxidation, promoting the observed increased plasma levels of these free fatty acids and glycerol. Interestingly, the essential n-3 free fatty acid C22:6 (DHA) showed a different response to the PCT (cluster 3), suggesting a DHA-specific metabolism. The plasma concentration of ketone bodies (3-hydroxybutanoic acid and acetonacetate) coincided with the availability of free fatty acids.

The cholesterol concentration decreased in response to PCT with subsequent recovery (cluster 3 ). The major portion of plasma cholesterol is carried in LDL lipoprotein particles. Increased insulin levels lead to activation of the enzyme lipoprotein lipase in adipose tissue, which in turn can lead to an increased clearance of lipoprotein particles and an inhibition of lipoprotein output from liver (Frayn 2010). Thus lowering of insulin and triglyceride levels at later time points may result in observed recovery of cholesterol levels (cluster 3). Apolipoprotein A1 showed a similar response as cholesterol, suggesting that plasma HDL particles showed similar behavior to LDL lipoprotein particles.

\subsubsection{Endocrine metabolism}

Testosterone and progesterone decreased postprandially, together with their precursor cholesterol. Similarly, sex hormone binding globulin (SHBG), the main transport binding protein for sex steroid hormones, decreased with subsequent recovery in response to the PCT (cluster 3). In line with this it has been shown that insulin can decrease SHBG synthesis in the liver (Pugeat et al. 2010), while testosterone levels decrease postprandially in men, followed by recovery after 2-3 h (Habito and Ball 2001).

Thyroid stimulating hormone (TSH) regulates the synthesis and secretion of the thyroid hormones which have an important role in the regulation of energy balance. Thyroxine binding globulin (TBG) is involved in the transport of thyroid hormones through blood. Both responded to the PCT with lower concentrations followed by a recovery (cluster 3), suggesting a decreased thyroid hormone production immediately after PCT. TSH concentrations have been reported to decrease immediately after ingestion of food (Kamat et al. 1995).

Cortisol is known to affect intermediary metabolism (glucose, fatty acid and amino acid metabolism) by binding to glucocorticoid receptors, counteracting the insulin action. Cortisol has metabolic effects on several tissues including stimulation of fat mobilization in adipose tissue, stimulation of gluconeogenesis, inhibition of the uptake of glucose by muscle and enhanced catabolism of muscle (Frayn 2010). Plasma cortisol concentrations decreased linearly in response to the PCT. The postprandial decrease of plasma cortisol concentrations upon a high fat diet was observed before (Volek et al. 2001).

\subsubsection{Energy metabolism}

The glycolysis intermediate pyruvate and the tricarboxylic acid cycle (TCA) intermediates citric acid and alpha-ketoglutaric acid showed temporary increased plasma levels upon PCT (cluster 2), whereas succinic acid showed only increased concentrations in the late phase of the time curve (cluster 6). This suggests that pyruvate, alpha-ketoglutarate and citric acid accumulate in the early phase and can not be efficiently metabolized for ATP conversion via TCA cycle, whereas succinic acid concentrations did not change in this early phase. The accumulation of pyruvate and alphaketoglutarate probably originates from surplus dietary amino acids that can be converted to these metabolites. These observations suggest that the TCA cycle has reached its optimum capacity in the early phase and recovers $3 \mathrm{~h}$ after PCT.

Plasma lactic acid showed linearly reduced concentrations (cluster 4), suggesting that ATP is solely aerobically produced in response to the PCT. The metabolite glycerol3-phosphate is synthesized from glycerol and can be used as substrate for glycolysis. However, this metabolite accumulated in plasma (cluster 5), suggesting that there is a surplus of NADH and that the process of oxidative phosphorylation may have reached its maximum capacity (Overkamp et al. 2002, in yeast). 


\subsubsection{Oxidative stress and inflammation}

It is known that oxidative stress is increased in postprandial state (Devaraj et al. 2008; Tsai et al. 2004). In our current study, this was reflected by increased plasma levels of myeloperoxidase (MPO) and matrix metallopeptidase 9 (MMP9) (cluster 5) and by decreased levels of the antioxidant uric acid (cluster 4). Postprandial increase of MPO and MMP9 was also observed by Spallarossa et al. (Spallarossa et al. 2008). It was suggested that these two enzymes contribute to impaired endothelial function in response to intake of a fat meal.

Also, food intake triggers a pro-inflammatory response, demonstrated e.g. by increased levels of IL-6 (Lundman et al. 2007; Poppitt et al. 2008). In response to the PCT, the IL-6 plasma concentration increased, together with levels of the inflammatory markers macrophage inflammatory protein 1 beta (MIP1-beta or CCL4) and extracellular newly identified RAGE-binding protein (EN-RAGE or S100A12). After an initial lag time, the concentration of IL-8 and macrophage derived cytokine (MDC or CCL22) also increased. A number of markers related to vascular health and extracellular matrix decreased in response to the PCT, at early time points (fibrinogen, connective tissue growth factor (CTGF), epidermal growth factor receptor (EGF-R), tenascin C-cluster 3) or continuously (factor VII, MMP-2, vascular cell adhesion molecule-1 (VCAM-1)—cluster 4). Concentrations of acute phase markers complement 3 and serum amyloid $\mathrm{P}$ decreased shortly after the PCT and recovered after $6 \mathrm{~h}$.

\subsection{Evaluation of the PCT in dietary intervention}

The rationale for the development of the PCT is that in dietary intervention studies, evaluation of the postprandial response would be more informative than quantification of non-perturbed conditions. In the section above, it was demonstrated that the postprandial response affects several metabolic pathways. In this section, we report on the analyses of differences in the postprandial time courses of proteins and metabolites after dietary intervention with an anti-inflammatory dietary mix (AIDM) compared to controls, and compare these findings with the differences found in fasting conditions. The effects of the AIDM on all plasma metabolites and proteins at fasting condition ( $\mathrm{t} 0$ ) has been reported earlier (Bakker et al. 2010). Also the detection of subtle dietary homeostatic (baseline) effects by repeated measures during the PCT were included in the former paper (Bakker et al. 2010) and although these have an added value for detection of subtle dietary effects by means of applying the PCT concept, they were currently considered to be out of scope.
The postprandial time courses of the plasma metabolites and proteins were quantified according to the parameters (AUCt, AUC-, AUC+, $\mathrm{T}_{\max }, \mathrm{T}_{\min }$ ) as described in Sect. 2. We identified 31 plasma parameters that responded differently to the AIDM intervention compared to the control (Table 1). Of these, 17 parameters were uniquely identified as responsive to the dietary intervention by applying the PCT and not in fasting conditions. The other 14 plasma parameters responded to the intervention during fasting ( $\mathrm{t} 0$ ) as well as postprandially. Out of the 31 metabolites and proteins that responded differently to the AIDM intervention compared to the control in response to PCT, 25 showed a significant difference in AUC values, while the remaining 6 differed in $\mathrm{T}_{\max }$ or $\mathrm{T}_{\min }$, i.e. they responded differently to the intervention by reaching maximum or minimum values at an earlier or later time point (Table 2). The differential postprandial responses between placebo and AIDM intervention are described in detail below.

\subsubsection{Amino acid metabolism}

Out of the 18 proteinogenic amino acids, 6 exhibited an altered response to the PCT after AIDM compared to the control. Isoleucine had a $12 \%$ increased AUC+ and a $13 \%$ increased AUCt, phenylalanine had a 19\% increased AUCt, proline had 54\% a decreased AUC - and valine had 16\% a increased AUC+ and 18\% increased a AUCt. All showed an increased concentration in plasma after AIDM intervention in comparison to control (Supplemental Fig. 1). AIDM intervention reduced the baseline fasting plasma concentrations of all 4 amino acids relative to control, but the differences between the groups disappeared at the postprandial state and similar postprandial plasma concentrations were found after $1 \mathrm{~h}\left(\mathrm{~T}_{\max }\right)$ and $6 \mathrm{~h}$ (results not shown). This suggests that the initial plasma concentration of these 4 amino acids regulates the amount of amino acids that is absorbed from the intestine in postprandial conditions.

The time required to reach the highest observed plasma concentration was significantly later in AIDM compared to placebo for the amino acids glutamine ( $\mathrm{T}_{\max }$ placebo $=1.3 \mathrm{~h}, \mathrm{~T}_{\max }$ AIDM $\left.=2.2 \mathrm{~h}\right)$ and tryptophan $\left(\mathrm{T}_{\max }\right.$ placebo $=1.3 \mathrm{~h}, \mathrm{~T}_{\max }$ AIDM $\left.=1.8 \mathrm{~h}\right)($ Table 2 and Supplemental Fig. 1).

\subsubsection{Exogenous metabolites}

Table 3 shows 4 metabolites of exogenous (i.e. not synthesized by human) origin that responded differently between AIDM and control exposed subjects to the PCT: free fatty acids $\mathrm{C} 22: 6$ and $\mathrm{C} 17: 0$, lactose and indole-3propionic acid. The essential omega-3 fatty acid DHA (C22:6) was present in the AIDM intervention causing 
Table 2 Identified metabolites and proteins that respond differentially between AIDM and placebo in response to PCT

\begin{tabular}{|c|c|c|c|}
\hline \# & Parameter & $\mathrm{AUC} / \mathrm{T}_{\max } / \mathrm{T}_{\min }$ & Fasting (t0) \\
\hline 1 & 2,4-Dihydroxybutanoic acid & $\mathbf{A U C}$ & No \\
\hline 2 & Lactose & AUC & No \\
\hline 3 & EGF-R & AUC & No \\
\hline 4 & Fibrinogen & AUC & No \\
\hline 5 & Glucagon & AUC & No \\
\hline 6 & $\mathbf{L H}$ & AUC & No \\
\hline 7 & ACE (CD143) & AUC & No \\
\hline 8 & CD40 & AUC & No \\
\hline 9 & von Willebrand factor & AUC & No \\
\hline 10 & Myeloperoxidase & $\mathbf{A U C}$ & No \\
\hline 11 & Thyroxine binding globulin & AUC & No \\
\hline 12 & $\operatorname{SM}(d 18: 1 / 22: 0)$ & $\mathbf{A U C}$ & No \\
\hline 13 & $\operatorname{SM}(d 17: 1 / 16: 0)$ & AUC & Yes $(\uparrow$ in AIDM) \\
\hline 14 & $\operatorname{SM}(d 17: 1 / 18: 0)$ & AUC & Yes $(\uparrow$ in AIDM) \\
\hline 15 & $\mathrm{SM}(\mathrm{d} 18: 1 / 16: 0)$ & AUC & Yes $(\uparrow$ in AIDM) \\
\hline 16 & Indole-3-propionic acid & AUC & Yes $(\uparrow$ in AIDM) \\
\hline 17 & Uric acid & AUC & Yes $(\downarrow$ in AIDM) \\
\hline 18 & C17:0 Fatty acid & AUC & Yes $(\downarrow$ in AIDM) \\
\hline 19 & C22:6 Fatty acid & AUC & Yes $(\uparrow$ in AIDM) \\
\hline 20 & Isoleucine & AUC & Yes $(\downarrow$ in AIDM) \\
\hline 21 & Phenylalanine & AUC & Yes $(\downarrow$ in AIDM) \\
\hline 22 & Proline & AUC & Yes $(\downarrow$ in AIDM) \\
\hline 23 & Valine & AUC & Yes $(\downarrow$ in AIDM) \\
\hline 24 & MDC & AUC & Yes $(\downarrow$ in AIDM) \\
\hline 25 & VCAM-1 & AUC & Yes $(\downarrow$ in AIDM) \\
\hline 26 & Glutamine & $\mathbf{T}_{\max }-\mathbf{L}$ & No \\
\hline 27 & Tryptophan & $\mathbf{T}_{\max }-\mathbf{L}$ & No \\
\hline 28 & 4-Hydroxyglutamate semialdehyde? & $\mathbf{T}_{\max }-\mathbf{L}$ & No \\
\hline 29 & Thyroid stimulating hormone & $\mathbf{T}_{\min }-\mathbf{E}$ & No \\
\hline 30 & $\operatorname{SM}(\mathrm{d} 18: 1 / 17: 0)$ & $\mathrm{T}_{\min }-\mathrm{E}$ & Yes $(\uparrow$ in AIDM) \\
\hline 31 & SHBG & $\mathrm{T}_{\min }-\mathrm{E}$ & Yes $(\uparrow$ in AIDM) \\
\hline
\end{tabular}

Bold plasma parameters are newly identified to respond to the dietary AIDM intervention

$\mathrm{L}=\mathrm{T}_{\max }$ or $\mathrm{T}_{\min }$ is reached significantly later in AIDM treated subjects compared to placebo treated subjects

$\mathrm{E}=\mathrm{T}_{\max }$ or $\mathrm{T}_{\min }$ is reached significant earlier in AIDM treated subjects compared to placebo treated subjects

$A U C$ area under curve (either AUC+, AUC- or AUCt)

increased fasting plasma concentrations in subjects on AIDM after the 5-week intervention. In response to the PCT plasma concentrations of DHA showed an increase in the early (up to $1 \mathrm{~h}$ ) and late response (from $3 \mathrm{~h}$ onwards) (Fig. 2a). Subjects on AIDM showed a higher release of DHA to plasma both in the early and late response compared to control subjects (Fig. 2a, 94\% increased AUC+ and a $140 \%$ increased AUCt). The fatty acid C17:0 originates primarily from animal products (dairy and meat) (Brevik et al. 2005). This exogenous fatty acid demonstrated decreased fasting concentrations in subjects on AIDM after the 5 weeks intervention (Table 2). In response to the PCT, plasma C17:0 showed increased concentrations in the early time response in subjects on AIDM, in contrast to the control group exhibiting reduced concentrations (Supplemental Fig. 1, 260\% increased AUCt). Indole-3-propionic acid is synthesized by gut microbiota (Wikoff et al. 2009). This metabolite showed increased fasting concentrations in subjects on AIDM, suggesting an effect of AIDM in the gastrointestinal tract. In response to PCT subjects on AIDM showed reduced plasma concentrations of indole-3-propionic acid in the late phase, whereas control subjects still showed elevated concentrations (Supplemental Fig. 1, 120\% increased 
Table 3 Nutritional value of $500 \mathrm{ml}$ postprandial challenge test formulation

\begin{tabular}{llll}
\hline Nutritional value & & Content & Energy \\
\hline Total & & $2945 \mathrm{~kJ}$ or $706 \mathrm{kcal}$ \\
Total protein & & $20.7 \mathrm{~g}$ & $11.7 \mathrm{E} \%$ \\
Total fat & Saturated fatty acids & $46.1 \mathrm{~g}$ & $58.7 \mathrm{E} \%$ \\
& Monounsaturated fatty acids & $27.1 \mathrm{~g}$ & $11.8 \mathrm{~g}$ \\
& Polyunsaturated fatty acids & $1.4 \mathrm{~g}$ & $2.1 \mathrm{~g}$ \\
& Cholesterol & $52.2 \mathrm{~g}$ & $29.6 \mathrm{E} \%$ \\
Total carbohydrates & & $42.3 \mathrm{~g}$ & \\
Total mono-disaccharides & & $0.1 \mathrm{~g}$ & \\
Total fiber & &
\end{tabular}

$g$ gram, $k J$ kilojoules, $k c a l$ kilocalories, $E \%$ energy percent

AUC-). Finally, lactose that likely derives from the PCT dairy shake showed a differential response between subjects on AIDM and placebo. Subjects on AIDM have a higher clearance rate of plasma lactose in comparison to subjects on placebo (Fig. 2b, 55\% decreased AUC-).

\subsubsection{Oxidative stress}

The PCT reveals additional effects of AIDM that may suggest reduced oxidative stress. Oxidative stress markers MDC (Fig. 2c, 52\% decreased AUC+ and a 162\% decreased AUCt) and myeloperoxidase (Supplemental Fig. 1, 25\% decreased AUC+ and a 27\% decreased AUCt) showed a delayed increase in response to the PCT after AIDM intervention suggesting a retarded or reduced oxidative stress response with AIDM. Consistent with this, AIDM intervention caused diminished reduction in response to PCT compared to control (Supplemental Fig. 1, $47 \%$ decreased AUC-), as well as $9 \%$ reduction of baseline concentrations of the antioxidant uric acid (Bakker et al. 2010).

\subsubsection{Inflammation}

The PCT reveals additional effects of AIDM that might suggest anti-inflammatory effects. The inflammatory markers CD40, VCAM1, fibrinogen and EGF-R show a more rapid reduction in response to PCT after AIDM intervention compared to placebo (Fig. 2d, e and Supplemental Fig. 1). CD40, VCAM1, fibrinogen and EGFR had a reduced AUC+ respectively of $25 \%, 66 \%, 80 \%$ and $52 \%$. VCAM1 and fibrinogen also had an increased AUCrespectively of $42 \%$ and $55 \%$ and AUCt respectively of $90 \%$ and $170 \%$ (negative AUCt in both conditions and both markers). Also levels of ACE (Supplemental Fig. 1) and von Willebrand factor (Fig. 2f) reduced in response to PCT because of AIDM intervention, whereas control intervention had no effect (fluctuations around baseline, 53\% decreased AUC+ for ACE, and 104\% increased AUCand $1291 \%$ decreased AUCt for von Willebrand factor). Interestingly, many of these factors are related to endothelial function (Chakrabarti et al. 2007; Constans and Conri 2006; Kakafika et al. 2007), supporting a potential beneficial effect of AIDM on vascular health (Bakker et al. 2010).

\subsubsection{Endocrine metabolism}

Glucagon (Fig. 2g) and luteinizing hormone (LH) (Supplemental Fig. 1), showed increased concentrations in response to PCT after AIDM intervention, whereas control intervention showed no alteration (44\% decreased AUCfor glucagon and $192 \%$ increased AUCt for LH). The plasma concentration of thyroid hormone transporter TBG showed a stronger reduction in subjects on AIDM compared to control (56\% increased AUC - and $87 \%$ increased AUCt (negative AUCt in both conditions)). Whereas the lowest observed plasma concentration was reached significantly earlier in AIDM compared to control for the sex hormone transporter SHBG and thyroid stimulating hormone (TSH) (Supplemental Fig. 1, SHBG; $\mathrm{T}_{\min }$ placebo $=3.2 \mathrm{~h}, \quad \mathrm{~T}_{\min } \quad \mathrm{AIDM}=2.2 \mathrm{~h} \quad$ and $\mathrm{TSH} ; \mathrm{T}_{\min }$ placebo $=3.1 \mathrm{~h}, \mathrm{~T}_{\min } \mathrm{AIDM}=2.4 \mathrm{~h}$ ). Thus, AIDM may influence endocrine metabolism, although it is difficult to interpret how these changes are related to health. The network in Fig. 3 shows connections between the outlined endocrine factors. Although there was no change in insulin sensitivity, glucose or insulin response to PCT between AIDM and control, there is a clear indication that AIDM influences endocrine metabolism. To what extent these changes are related to health remains to be elucidated. 

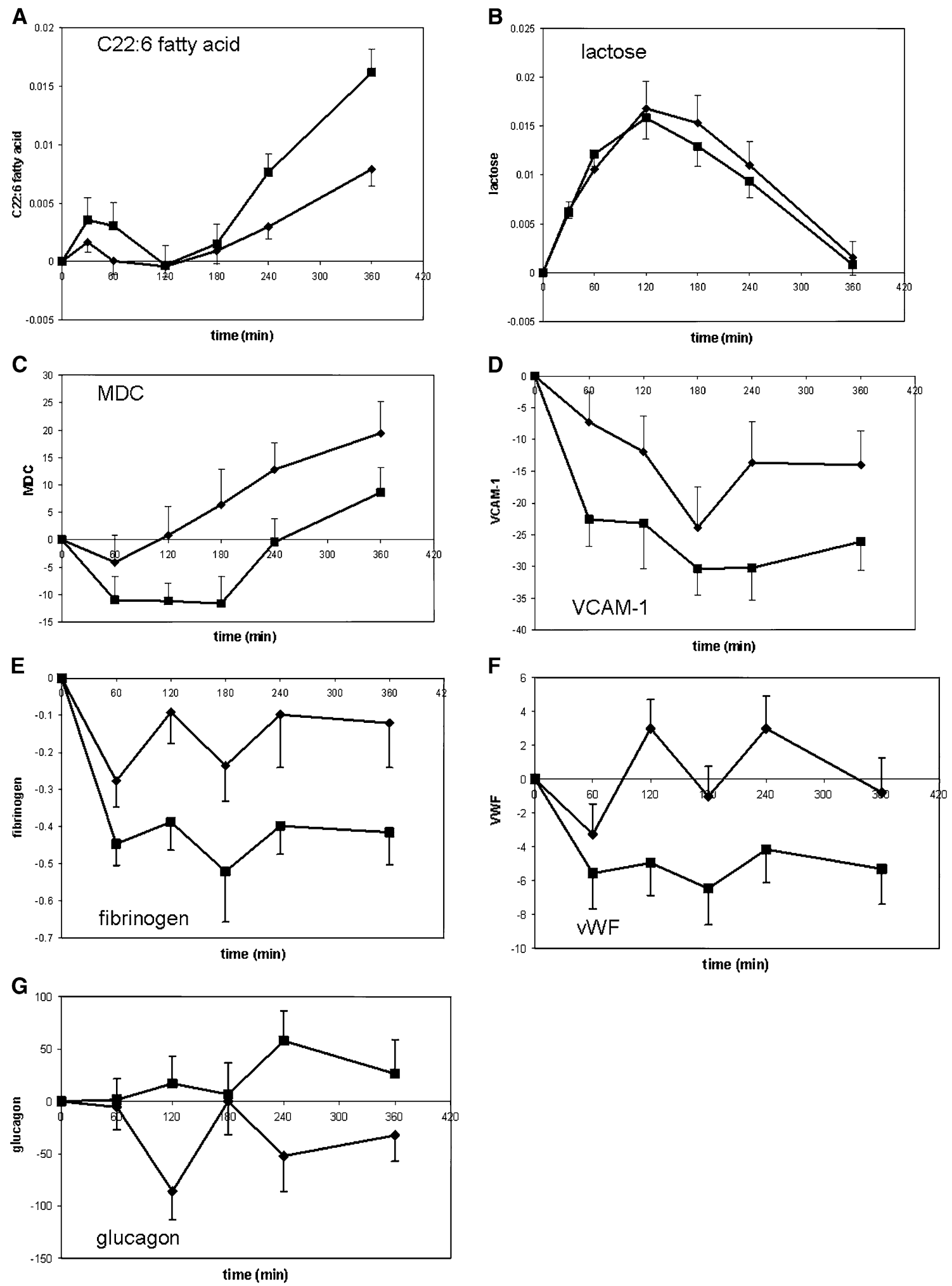

Fig. 2 Responses to PCT after AIDM and placebo intervention. Figures show mean $( \pm$ SEM) difference versus t0 for each time point. Differences versus t0 were calculated for 35 (metabolites) or 33 subjects (proteins). Diamond indicates placebo intervention and square indicates AIDM intervention. $M D C$ Macrophage-derived

chemokine, VCAM-1 vascular cell adhesion protein 1, $v W F$ von Willebrand factor. $Y$-axis are relative concentrations for metabolites C22:6 fatty acid and lactose; for proteins units for difference in concentration (compared to t0) are as follows: MDC pg/ml, VCAM$1 \mathrm{ng} / \mathrm{ml}$, fibrinogen $\mathrm{mg} / \mathrm{ml}, \mathrm{vWF} \mu \mathrm{g} / \mathrm{ml}$ and glucagon $\mathrm{pg} / \mathrm{ml}$ 
Fig. 3 Network showing connections between endocrine factors with a differential response to PCT after AIDM intervention compared to placebo (MetaCore, network option auto expand)

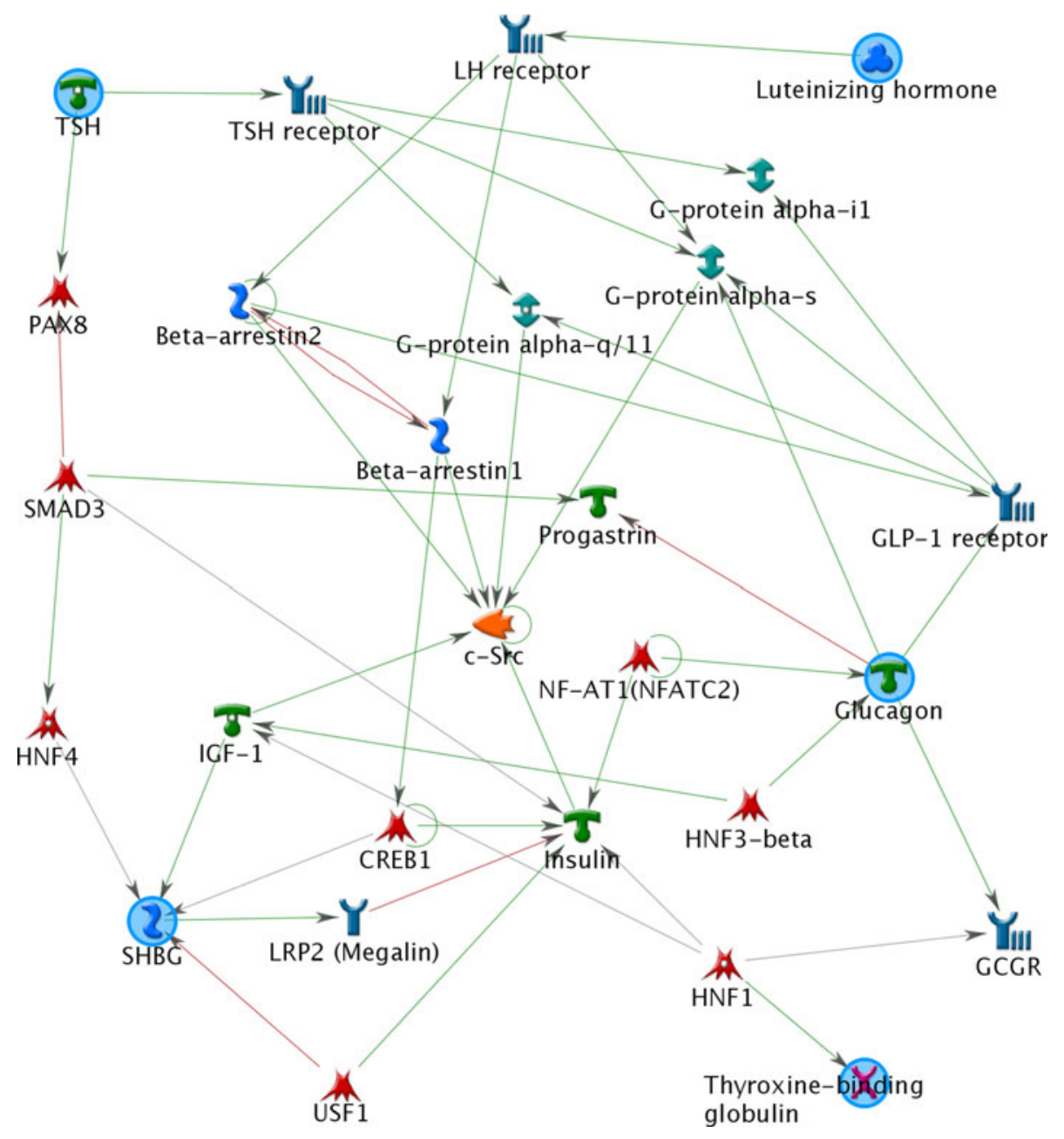

It is difficult to compare our results with other studies, because no standardized PCT is available. The meal challenges differ in energy content from 250 to $2500 \mathrm{kcal}$, from 50 to $100 \mathrm{E} \%$ fat, $0-50 \mathrm{E} \%$ carbohydrates, and 0 to $20 \mathrm{E} \%$ protein (Paglialunga and Cianflone 2007). Moreover these PCTs also differ in composition, solid versus liquid, and amount (Cianflone et al. 2008).

\section{Concluding remarks}

The PCT affected carbohydrate, amino acid, lipid and lipoprotein metabolism. Furthermore, the processes energy metabolism, oxidative stress, inflammation and endocrine response reacted to the PCT. New observations included the different response of n-3 fatty acid DHA compared to other long chain free fatty acids and that a PCT may affect indicators of bone metabolism.

Assessment of metabolic changes due to a dietary intervention revealed by the PCT resulted in 31 plasma parameters that showed a differential response to the PCT after AIDM compared to placebo intervention. More than $50 \%$ of these were uniquely changed when applying the PCT. Other parameters changed both in perturbed and nonperturbed conditions, which offered additional insight in biological responses. For example, now we showed that the intervention reduced the short term, acute vascular inflammatory and oxidative stress response, in addition to the effect on vascular health and oxidative stress in fasted (homeostatic) conditions (Bakker et al. 2010).

This nutrigenomics based PCT showed the relevance of metabolic perturbation for quantification of subtle phenotypic changes. Applying challenge tests and measuring the integrated responses should be further developed as a tool to quantify and define optimal health.

Acknowledgments We express our gratitude to the volunteers participating in the study, staff of the Metabolic Research Unit, laboratories and groups contributing to the study and analyses, our colleagues contributing in designing the experiment and Professor Christian Drevon (University of Oslo, department of Nutrition) for critical reading of the manuscript. This study was supported by TNO. TNO is a member of the Nutrigenomics Organization (www.nugo.org). 
Open Access This article is distributed under the terms of the Creative Commons Attribution Noncommercial License which permits any noncommercial use, distribution, and reproduction in any medium, provided the original author(s) and source are credited.

\section{References}

Bakker, G. C. M., Van Erk, M. J., Pellis, L., Wopereis, S., Rubingh, C. M., Cnubben, N. H. P., et al. (2010). An antiinflammatory dietary mix modulates inflammation and oxidative and metabolic stress in overweight men: A nutrigenomics approach. American Journal of Clinical Nutrition, 91(4), 1044-1059.

Bijlsma, S., Bobeldijk, I., Verheij, E. R., Ramaker, R., Kochhar, S., Macdonald, I. A., et al. (2006). Large-scale human metabolomics studies: A strategy for data (pre-) processing and validation. Analytical Chemistry, 78(2), 567-574.

Brevik, A., Veierod, M. B., Drevon, C. A., \& Andersen, L. F. (2005). Evaluation of the odd fatty acids 15:0 and 17:0 in serum and adipose tissue as markers of intake of milk and dairy fat. European Journal of Clinical Nutrition, 59(12), 1417-1422.

Carroll, M. F., \& Schade, D. S. (2003). Timing of antioxidant vitamin ingestion alters postprandial proatherogenic serum markers. Circulation, 108(1), 24-31.

Cawston, T., \& Young, D. (2010). Proteinases involved in matrix turnover during cartilage and bone breakdown. Cell and Tissue Research, 339(1), 221-235.

Chakrabarti, S., Blair, P., \& Freedman, J. E. (2007). CD40-40L signaling in vascular inflammation. Journal of Biological Chemistry, 282(25), 18307-18317.

Cianflone, K., Paglialunga, S., \& Roy, C. (2008). Intestinally derived lipids: Metabolic regulation and consequences-An overview. Atherosclerosis Supplements, 9(2), 63-68.

Constans, J., \& Conri, C. (2006). Circulating markers of endothelial function in cardiovascular disease. Clinica Chimica Acta, 368(1-2), 33-47.

Devaraj, S., Wang-Polagruto, J., Polagruto, J., Keen, C. L., \& Jialal, I. (2008). High-fat, energy-dense, fast-food-style breakfast results in an increase in oxidative stress in metabolic syndrome. Metabolism, 57(6), 867-870.

Elliott, R., Pico, C., Dommels, Y., Wybranska, I., Hesketh, J., \& Keijer, J. (2007). Nutrigenomic approaches for benefit-risk analysis of foods and food components: Defining markers of health. British Journal of Nutrition, 98(6), 1095-1100.

Esposito, K., Nappo, F., Giugliano, F., Di, P. C., Ciotola, M., Barbieri, M., et al. (2003). Meal modulation of circulating interleukin 18 and adiponectin concentrations in healthy subjects and in patients with type 2 diabetes mellitus. American Journal of Clinical Nutrition, 78(6), 1135-1140.

Frayn, K. N. (2010). Metabolic regulation-A human perspective. Blackwell Publishers.

Ghanim, H., Abuaysheh, S., Sia, C. L., Korzeniewski, K., Chaudhuri, A., Fernandez-Real, J. M., et al. (2009). Increase in plasma endotoxin concentrations and the expression of toll-like receptors and suppressor of cytokine signaling-3 in mononuclear cells after a high-fat, high-carbohydrate meal. Diabetes Care, 32(12), 2281-2287.

Goldtein, M. S., Henry, W. L., Huddlestun, B., \& Levine, R. (1953). Action of insulin on transfer of sugars across cell barriers; common chemical configuration of substances responsive to action of the hormone. American Journal of Physiology, 173(2), 207-211.

Habito, R. C., \& Ball, M. J. (2001). Postprandial changes in sex hormones after meals of different composition. Metabolism, $50(5), 505-511$.
Jackson, K. G., Armah, C. K., \& Minihane, A. M. (2007). Meal fatty acids and postprandial vascular reactivity. Biochemical Society Transactions, 035(3), 451-453.

Kakafika, A. I., Liberopoulos, E. N., \& Mikhailidis, D. P. (2007). Fibrinogen: A predictor of vascular disease. Current Pharmaceutical Design, 13(16), 1647-1659.

Kamat, V., Hecht, W. L., \& Rubin, R. T. (1995). Influence of meal composition on the postprandial response of the pituitary-thyroid axis. European Journal of Endocrinology, 133(1), 75-79.

Koek, M., van der Kloet, F., Kleemann, R., Kooistra, T., Verheij, E., \& Hankemeier, T. (2011). Semi-automated non-target processing in GC $\times$ GC-MS metabolomics analysis: Applicability for biomedical studies. Metabolomics, 7(1), 1-14.

Lopez-Miranda, J., Williams, C., \& Lairon, D. (2007). Dietary, physiological, genetic and pathological influences on postprandial lipid metabolism. British Journal of Nutrition, 98(3), 458-473.

Lundman, P., Boquist, S., Samnegsrd, A., Bennermo, M., Held, C., Ericsson, C. G., et al. (2007). A high-fat meal is accompanied by increased plasma interleukin-6 concentrations. Nutrition, Metabolism and Cardiovascular Diseases, 17(3), 195-202.

Margioris, A. N. (2009). Fatty acids and postprandial inflammation. Current Opinion in Clinical Nutrition \& Metabolic Care, 12(2), 129-137.

Minisola, S., Antonelli, R., \& Mazzuoli, G. (1985). Clinical significance of free plasma hydroxyproline measurement in metabolic bone disease. Journal of Clinical Chemistry \& Clinical Biochemistry, 23(9), 515-519.

Nakatsuji, H., Kishida, K., Kitamura, T., Nakajima, C., Funahashi, T., \& Shimomura, I. (2010). Dysregulation of glucose, insulin, triglyceride, blood pressure, and oxidative stress after an oral glucose tolerance test in men with abdominal obesity. Metabolism: Clinical and Experimental, 59(4), 520-526.

Nappo, F., Esposito, K., Cioffi, M., Giugliano, G., Molinari, A. M., Paolisso, G., et al. (2002). Postprandial endothelial activation in healthy subjects and in type 2 diabetic patients: Role of fat and carbohydrate meals. Journal of the American College of Cardiology, 39(7), 1145-1150.

Neri, S., Signorelli, S. S., Torrisi, B., Pulvirenti, D., Mauceri, B., Abate, G., et al. (2005). Effects of antioxidant supplementation on postprandial oxidative stress and endothelial dysfunction: A single-blind, 15-day clinical trial in patients with untreated type 2 diabetes, subjects with impaired glucose tolerance, and healthy controls. Clinical Therapeutics, 27(11), 1764-1773.

Nowatzky, J., Howard, R., Pillinger, M. H., \& Krasnokutsky, S. (2010). The role of uric acid and other crystals in osteoarthritis. Current Rheumatology Reports, 12(2), 42-148.

Overkamp, K. M., Bakker, B. M., Kotter, P., Luttik, M. A., van Dijken, J. P., \& Pronk, J. T. (2002). Metabolic engineering of glycerol production in Saccharomyces cerevisiae. Applied and Environmental Microbiology, 68(6), 2814-2821.

Paglialunga, S., \& Cianflone, K. (2007). Regulation of postprandial lipemia: An update on current trends. Applied Physiology, Nutrition, and Metabolism, 32(1), 61-75.

Parfitt, A. M. (2002). Targeted and nontargeted bone remodeling: Relationship to basic multicellular unit origination and progression. Bone, 30(1), 5-7.

Poppitt, S. D., Keogh, G. F., Lithander, F. E., Wang, Y., Mulvey, T. B., Chan, Y. K., et al. (2008). Postprandial response of adiponectin, interleukin-6, tumor necrosis factor-[alpha], and C-reactive protein to a high-fat dietary load. Nutrition, 24(4), 322-329.

Pugeat, M., Nader, N., Hogeveen, K., Raverot, G., Dechaud, H., \& Grenot, C. (2010). Sex hormone-binding globulin gene expression in the liver: Drugs and the metabolic syndrome. Molecular and Cellular Endocrinology, 316(1), 53-59. 
Riches, P. L., \& Ralston, S. H. (2010). Recent insights into the biology of bone turnover. Journal of the Royal College of Physicians of Edinburgh, 40(1), 66-69.

Schett, G., Kiechl, S., Bonora, E., Zwerina, J., Mayr, A., Axmann, R., et al. (2009). Vascular cell adhesion molecule 1 as a predictor of severe osteoarthritis of the hip and knee joints. Arthritis and Rheumatism, 60(8), 2381-2389.

Shaham, O., Wei, R., Wang, T. J., Ricciardi, C., Lewis, G. D., Vasan, R. S., et al. (2008). Metabolic profiling of the human response to a glucose challenge reveals distinct axes of insulin sensitivity. Molecular Systems Biology, 4, 214-222.

Spallarossa, P., Garibaldi, S., Barisione, C., Ghigliotti, G., Altieri, P., Tracchi, I., et al. (2008). Postprandial serum induces apoptosis in endothelial cells: Role of polymorphonuclear-derived myeloperoxidase and metalloproteinase-9 activity. Atherosclerosis, 198(2), 458-467.

Tsai, W. C., Li, Y. H., Lin, C. C., Chao, T. H., \& Chen, J. H. (2004). Effects of oxidative stress on endothelial function after a high-fat meal. Clinical Science, 106(3), 315-319.

van der Kloet, F. M., Bobeldijk, I., Verheij, E. R., \& Jellema, R. H. (2009). Analytical error reduction using single point calibration for accurate and precise metabolomic phenotyping. Journal of Proteome Research, 8(11), 5132-5141.

van Eijk, H. M., Deutz, N. E., Wagenmakers, A. J., \& Soeters, P. B. (1990). 3-Methylhistidine determined in plasma by "high-performance" lipid chromatography. Clinical Chemistry, 36(3), 556-559.

van Ommen, B., Keijer, J., Heil, S. G., \& Kaput, J. (2009). Challenging homeostasis to define biomarkers for nutrition related health. Molecular Nutrition \& Food Research, 53(7), 795-804.

van Ommen, B., Keijer, J., Kleemann, R., Elliott, R., Drevon, C., McArdle, H., et al. (2008). The challenges for molecular nutrition research 2: Quantification of the nutritional phenotype. Genes \& Nutrition, 3(2), 51-59.

Volek, J. S., Gomez, A. L., Love, D. M., Avery, N. G., Sharman, M. J., \& Kraemer, W. J. (2001). Effects of a high-fat diet on postabsorptive and postprandial testosterone responses to a fat-rich meal. Metabolism, 50(11), 1351-1355.
Walsh, J. S., \& Henriksen, D. B. (2010). Feeding and bone. Archives of Biochemistry and Biophysics, 503(1), 11-19.

Wang, H., \& Eckel, R. H. (2009). Lipoprotein lipase: From gene to obesity. American Journal of Physiology-Endocrinology and Metabolism, 297(2), E271-E288.

Waterworth, D. M., Talmud, P. J., Luan, J., Flavell, D. M., Byrne, C. D., Humphries, S. E., et al. (2003). Variants in the APOC3 promoter insulin responsive element modulate insulin secretion and lipids in middle-aged men. Biochimica et Biophysica Acta (BBA) - Molecular Basis of Disease, 1637(3), 200-206.

Wikoff, W. R., Anfora, A. T., Liu, J., Schultz, P. G., Lesley, S. A., Peters, E. C., et al. (2009). Metabolomics analysis reveals large effects of gut microflora on mammalian blood metabolites. Proceedings of the National Academy of Sciences of the United States of America, 106(10), 3698-3703.

Wopereis, S., Rubingh, C. M., Van Erk, M. J., Verheij, E. R., van Vliet, V. T., Cnubben, N. H., et al. (2009). Metabolic profiling of the response to an oral glucose tolerance test detects subtle metabolic changes. PLoS One, 4(2), e4525.

Wybranska, I., Malczewska-Malec, M., Niedbal, S., Naskalski, J. W., \& Dembinska-Kiec, A. (2003). The TNF-alpha gene NcoI polymorphism at position-308 of the promoter influences insulin resistance, and increases serum triglycerides after postprandial lipaemia in familiar obesity. Clinical Chemistry and Laboratory Medicine, 41(4), 501-510.

Wyss, M., \& Kaddurah-Daouk, R. (2000). Creatine and creatinine metabolism. Physiological Reviews, 80(3), 1107-1213.

Zhao, X., Peter, A., Fritsche, J., Elcnerova, M., Fritsche, A., Haring, H. U., et al. (2009). Changes of the plasma metabolome during an oral glucose tolerance test: Is there more than glucose to look at? American Journal of Physiology-Endocrinology and Metabolism, 296(2), E384-E393.

Zivkovic, A., Wiest, M., Nguyen, U., Nording, M., Watkins, S., \& German, J. (2008). Assessing individual metabolic responsiveness to a lipid challenge using a targeted metabolomic approach. Metabolomics, 5(2), 209-218. 